\title{
Combined extracranial and intracranial approach for resection of dermoid cyst of the sphenoid bone with a cutaneous sinus tract across the frontal branch of the facial nerve
}

\author{
Naohiro Ishii ${ }^{1}$, \\ Emi Fukazawa ${ }^{2}$, \\ Tomoko Aoki², \\ Kazuo Kishi ${ }^{2}$ \\ ${ }^{1}$ Department of Plastic and \\ Reconstructive Surgery, International \\ University of Health and Welfare \\ Hospital, Nasushiobara; \\ ${ }^{2}$ Department of Plastic and \\ Reconstructive Surgery, Keio University, \\ Tokyo, Japan
}

\begin{abstract}
Frontotemporal dermoid cysts with a cutaneous sinus tract in the sphenoid bone are rarely found, and furthermore, the spreading of these cysts across the frontal branch of the facial nerve has not been reported. Herein, we present a 5-year-old case of a dermoid cyst successfully resected with preservation of this nerve using a combined extracranial and intracranial approach. This approach is recommended for a safe and radical resection of the lesion and for securing an aesthetic outcome.
\end{abstract}

Keywords: Dermoid cyst / Facial nerve / Skull

\section{INTRODUCTION}

Craniofacial dermoid cysts are congenital or acquired developmental malformations containing the skin, and its appendages, such as the hair follicles, and sebaceous and sweat glands are commonly found in the lateral orbital region [1,2]. Dermoid cysts of the cranial bones are thought to develop from the entrapment of embryonic ectoderm along the cranial suture lines during fetal development, and those developing in the sphenoid bone are rare (3\% of all dermoid cysts of craniofacial bone). In addition, they tend to be difficult to be found during early periods, since they are deep, slow-growing lesions [3,4].

They develop with a cutaneous sinus tract in rare cases [5], and dermoid cysts with a cutaneous sinus tract in sphenoid bone have been reported to date in only a few cases $[3,4,6,7]$. In

Correspondence: Naohiro Ishii

Department of Plastic and Reconstructive Surgery, International University of Health and Welfare Hospital, 537-3 Iguchi, Nasushiobara 329-2763, Japan

E-mail: ishinao0916@gmail.com

Received October 25, 2018 / Revised January 27, 2019 / Accepted February 1, 2019 such cases, an approach aimed to preserve the frontal branch of facial nerve, and to achieve a radical but aesthetic resection of the extracranial and intracranial lesion may be required, however, to the best of our knowledge, no report has been reported to date.

Herein, we present a rare case of a frontotemporal dermoid cyst in a child with a cutaneous sinus tract across the frontal branch of the facial nerve and deep extension through the sphenoid bone. The cyst was successfully resected with preservation of the nerve using two approaches (lateral orbital skin and temporal hair-bearing area). We achieved good results, and the approach was radically curative and aesthetically effective.

\section{CASE REPORT}

A 5-year-old boy had a congenital subcutaneous tumor in the frontotemporal region. Three years prior, it was swollen due to infection several times, and it was drained each time as an infectious atheroma at a local clinic. Thereafter, he was referred to 
our hospital due to repeated infection. At the initial visit, the tumor was associated with pus and infection, and its subcutaneous size was $1.5 \mathrm{~cm} \times 1.5 \mathrm{~cm}$. The patient had no significant past medical history, except for the tumor.

Computed tomography (CT) demonstrated subcutaneous cystic lesions as low-density areas and the lesion of sphenoid bone as a bony defect (Fig. 1). Magnetic resonance imaging (MRI) demonstrated a cystic lesion with a cutaneous sinus tract ranging from the subcutaneous tissue to temporal fossa, as a low signal in the T1-weighted image and as a high signal in the T2-weighted image (Fig. 2). The CT and MRI findings were suggestive of a dermoid cyst of the sphenoid bone with a cutaneous sinus tract. Furthermore, the images and location of the skin lesion suggested that this tract traversed medially across the course of the frontal branch of the facial nerve since this nerve locates between the line running from $5 \mathrm{~cm}$ above the upper border and lateral position of eyebrow to the tragus and the line running from the median height and lateral position of eyebrow to the earlobe base $[8,9]$.

After controlling the infection, tumorectomy was performed, in cooperation with a neurosurgeon, under general anesthesia. We planned resection of the subcutaneous tumor above the cranial bone using a tumor marginal incision and the sphenoid bone lesion using an incision in the temporal hair-bearing area, which marked the course of the frontal branch of the facial nerve as described above (Fig. 3). The cutaneous sinus tract was carefully dissected thorough the skin, subcutaneous tissue, and

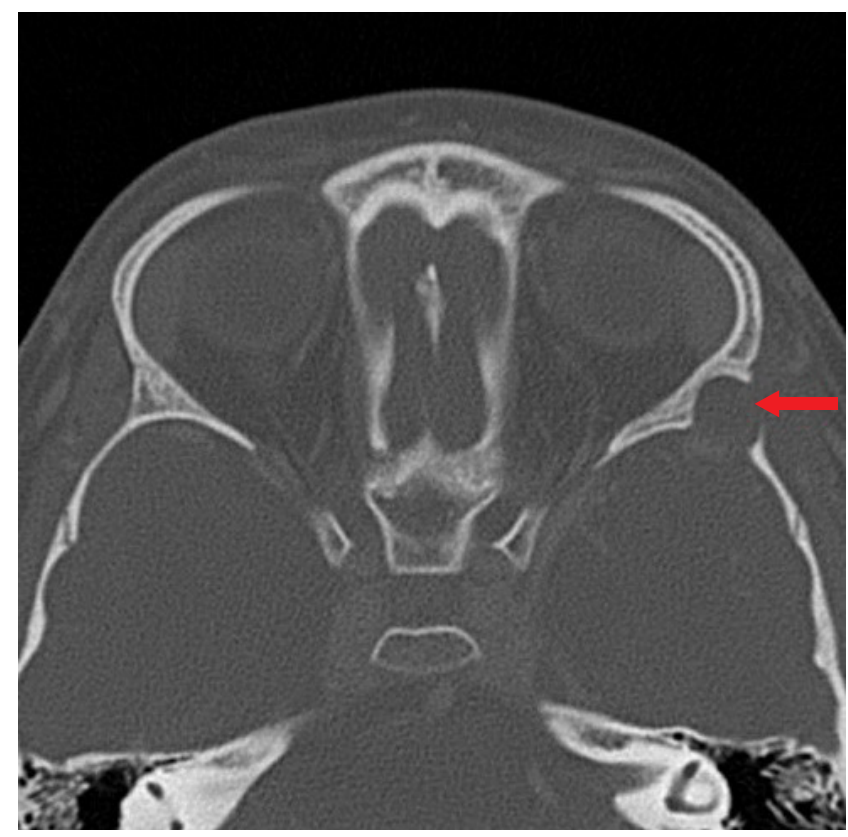

Fig. 1. Preoperative computed tomography image of the tumor. The lesion of the sphenoid bone demonstrated as a bony defect (red arrow). temporal muscle with the preservation of the facial nerve (Fig. 4). Subsequently, the temporal hair-bearing area incision and underlying periosteum secured a surgical field enough to resect the lesion of the sphenoid bone. The sphenoid periosteum was incised, and subsequently, the sphenoid bone around its fistula was shaved off and the sphenoid cyst was exposed. The sphe-

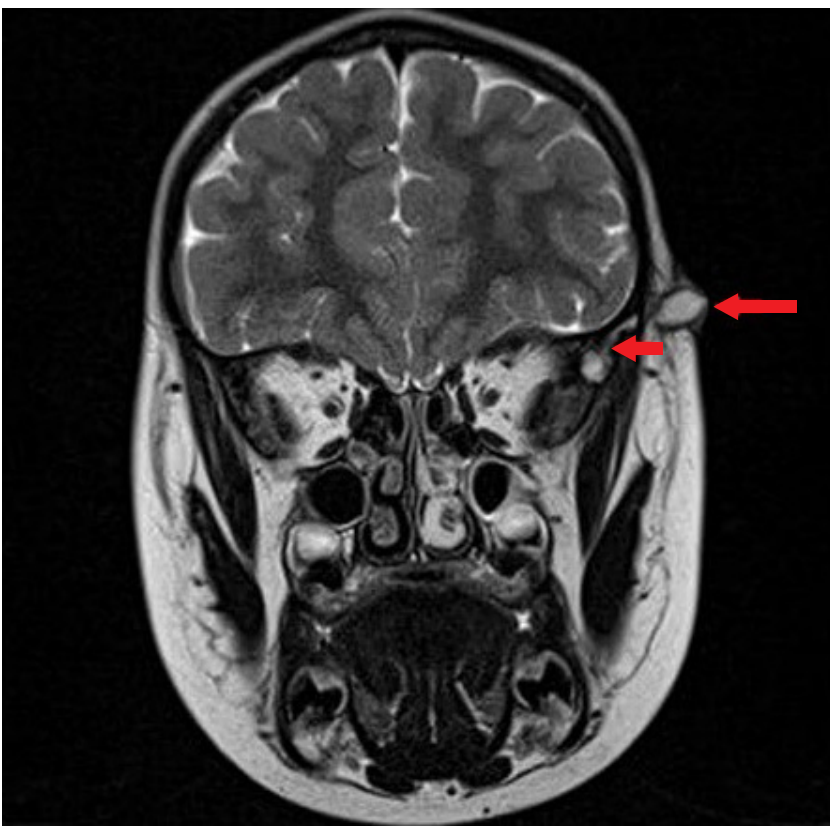

Fig. 2. Preoperative magnetic resonance image of the tumor. Cystic lesion with a cutaneous sinus tract ranging from subcutaneous tissue to temporal fossa (red arrows).

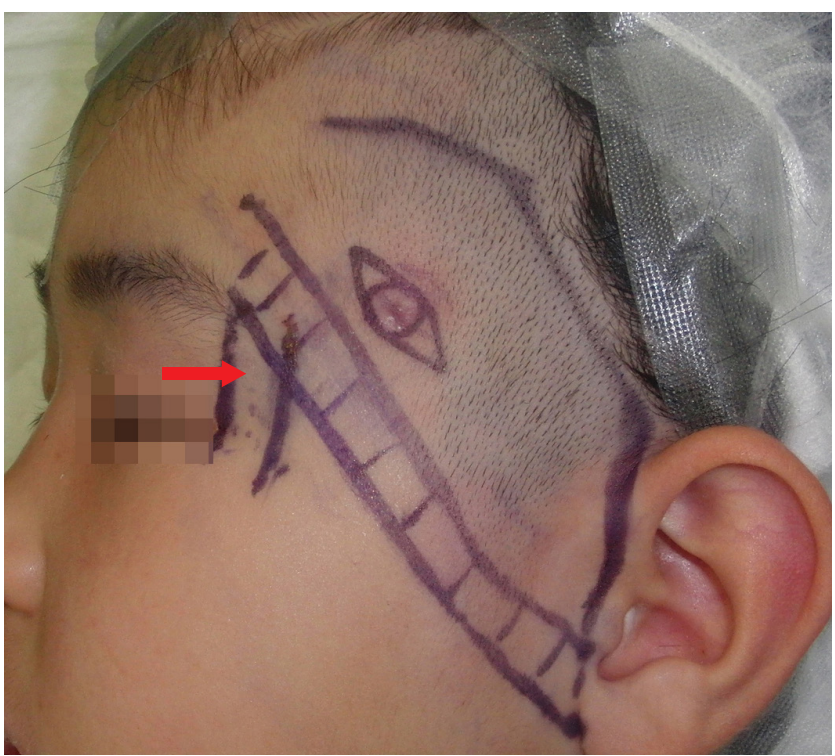

Fig. 3. Skin incision for resection of the subcutaneous tumor above the cranial bone, temporal hair-bearing area incision for resection of the lesion of sphenoid bone. Ladder-type line shows the course of the frontal branch of the facial nerve and a red arrow shows the lateral orbital edge. 


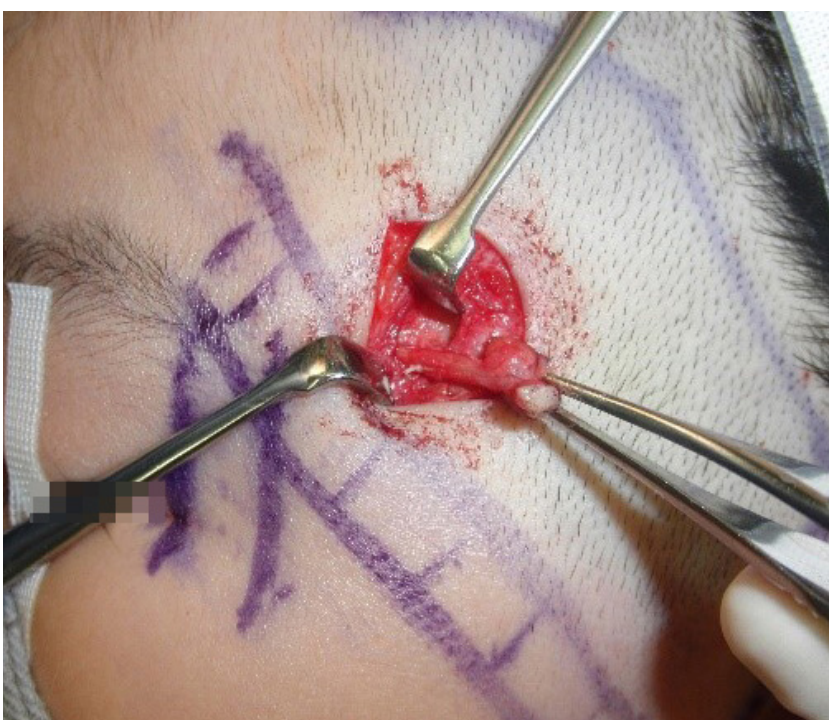

Fig. 4. Intraoperative photograph of the skin tumor and cutaneous sinus tract. These were separated from surrounded tissue, with a surgical knife, through the temporal muscle, with no exposure of the facial nerve, thus preserving it.

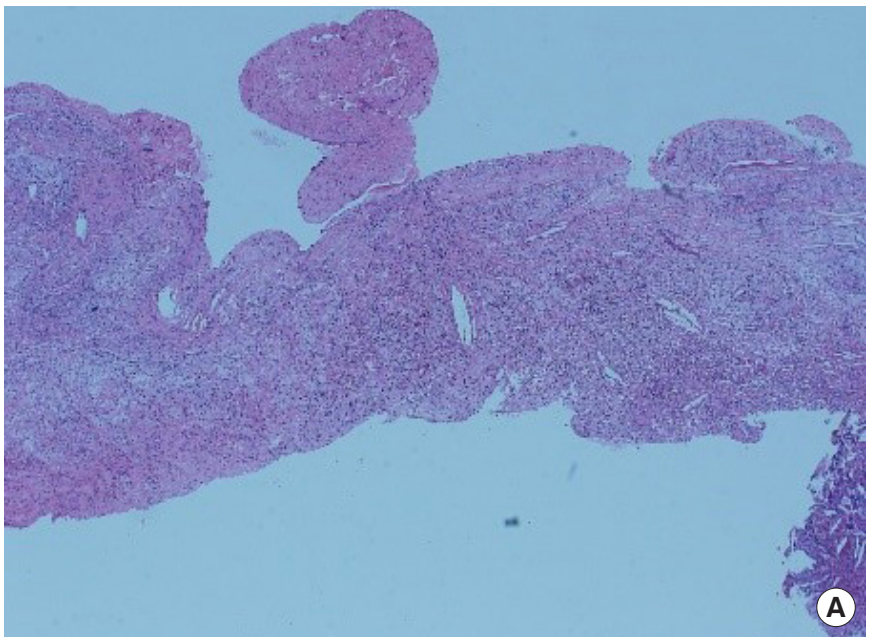

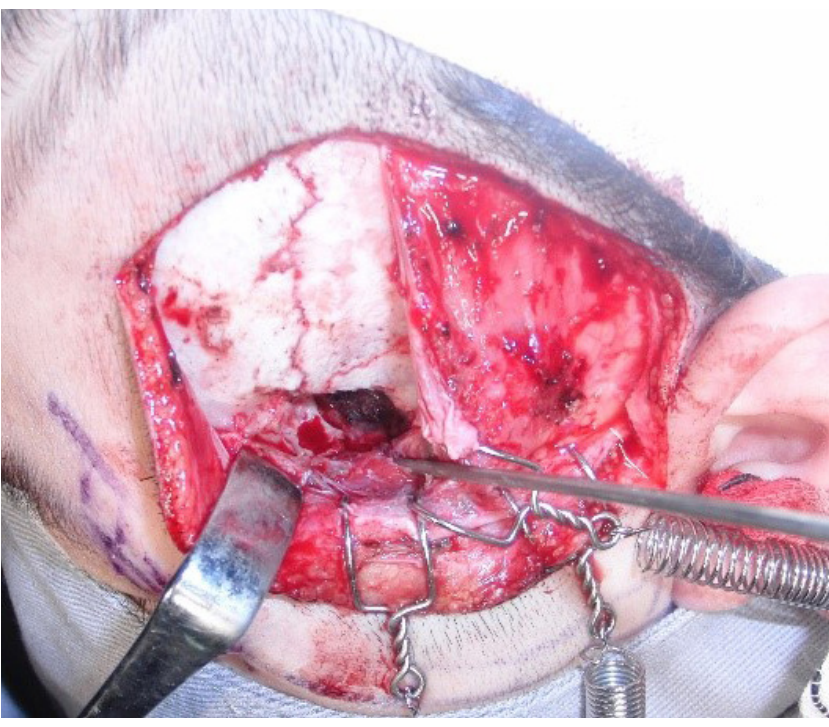

Fig. 5. Intraoperative photograph after resection of the lesion of sphenoid bone without rupture of dura and superficial electrocautery to dura.

Fig. 6. (A, B) Histopathological image of the tumor. Collection of foamy macrophages with hair and cholesterol granuloma indicative of dermoid cyst (H\&E: $A, \times 4$ and $B, \times 40)$.

noid cyst was in contact with the dura; however, it could be resected without rupture of the dura. Additionally, superficial electrocautery of the dura was performed to prevent recurrence (Fig. 5). Following saline irrigation and replacement of suction drain, skin closure was performed.

Surgical pathology demonstrated a sphenoid bone dermoid cyst with a cutaneous sinus tract (Fig. 6). No complications occurred during the perioperative and postoperative period and follow-up for 5 years showed no recurrence and a good aesthetic result (Fig. 7).

\section{DISCUSSION}

Dermoid cysts of the cranial bone rarely develop with a cutaneous sinus tract, and subcutaneous infection in $15 \%$ of such cases often make patients recognize its occurrence [5]. Their main lesion located in the deep layer cannot be found by visual examination and palpation, and it sometimes destroys the cranial bone and invades in to the dura $[2,10,11]$. Therefore, surgical removal in this region must be based on preoperative imaging.

CT can demonstrate dermoid cysts in low-density areas and the lesions of cranial bone are seen as bony defects [12]. MRI can demonstrate dermoid cysts as a low signal in a T1-weighted 


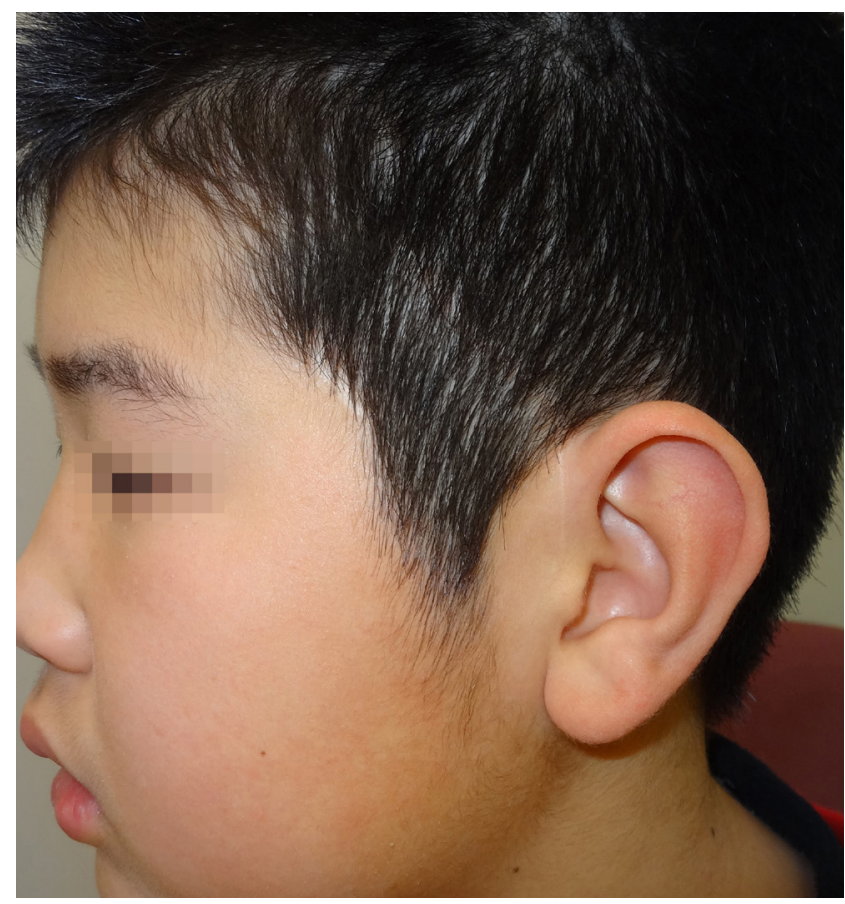

Fig. 7. Postoperative photograph after 5 years showing no recurrence and good aesthetic results.

image and as a high signal in a T2-weighted image, and is suitable for detecting a cutaneous sinus tract [13]. Therefore, both $\mathrm{CT}$ and MRI are necessary for preoperative imaging in cases such as ours.

Complete resection of dermoid cysts of cranial bone with a cutaneous sinus tract often requires elaborate plans. A cutaneous sinus tract sometimes becomes complicated due to repeated infections, and the usefulness of methylene blue staining in diagnosis of a complicated tract has been reported $[14,15]$. However, in the present case, MRI demonstrated only one cutaneous sinus tract, and therefore, it could be exposed by using surgical bougie.

For the resection of large and deep cranial dermoid cysts, a wide surgical field should be secured in the point of radicality and safety. Large cranial defect needs augmentation with artificial bone, and definite invasion in the dura needs its resection and reconstruction. In the present case, cranial reconstruction was not needed since cranial defects were comparatively small and the temporal muscle was preserved. Furthermore, the tumor could be resected without rupture of the dura, and only superficial electrocautery to the dura was performed to prevent recurrence.

Frontotemporal dermoid cysts with a cutaneous sinus tract in sphenoid bone have been reported to date in only a few cases, however, the spreading of these lesions across the frontal branch of the facial nerve has not been reported. An appropri- ate approach is required to ensure preservation of this nerve, safety, radicality, and aesthetic outcome.

With these considerations, we completely resected the cutaneous tumor with a sinus using minimum skin incision and the cranial tumor with securing a wide surgical field by a hair-bearing area incision. Furthermore, long postoperative period with no recurrence and a good aesthetic outcome have been observed.

\section{NOTES}

\section{Conflict of interest}

No potential conflict of interest relevant to this article was reported.

\section{Ethical approval}

The study was performed in accordance with the principles of the Declaration of Helsinki. Written informed consent was obtained.

\section{Patient consent}

The patient provided written informed consent for the publication and the use of his images.

\section{ORCID}

Naohiro Ishii https://orcid.org/0000-0002-0906-5623

Emi Fukazawa https://orcid.org/0000-0003-2727-6544

Tomoko Aoki https://orcid.org/0000-0003-2191-7371

Kazuo Kishi https://orcid.org/0000-0002-4298-9828

\section{REFERENCES}

1. Brownstein MH, Helwig EB. Subcutaneous dermoid cysts. Arch Dermatol 1973;107:237-9.

2. Kronish JW, Dortzbach RK. Upper eyelid crease surgical approach to dermoid and epidermoid cysts in children. Arch Ophthalmol 1988;106:1625-7.

3. Hong SW. Deep frontotemporal dermoid cyst presenting as a discharging sinus: a case report and review of literature. $\mathrm{Br} \mathrm{J}$ Plast Surg 1998;51:255-7.

4. Honig JF. A de novo discharging sinus of the fronto-orbital suture: a rare presentation of a dermoid cyst. J Craniofac Surg 1998;9:536-8.

5. Chawda SJ, Moseley IF. Computed tomography of orbital dermoids: a 20-year review. Clin Radiol 1999;54:821-5.

6. Yan C, Low DW. A rare presentation of a dermoid cyst with draining sinus in a child: case report and literature review. Pediatr Dermatol 2016;33:e244-8. 
7. Scolozzi P, Lombardi T, Jaques B. Congenital intracranial frontotemporal dermoid cyst presenting as a cutaneous fistula. Head Neck 2005;27:429-32.

8. Davis RA, Anson BJ, Budinger JM, Kurth LR. Surgical anatomy of the facial nerve and parotid gland based upon a study of 350 cervicofacial halves. Surg Gynecol Obstet 1956;102:385-412.

9. May M. Anatomy of the facial nerve (spatial orientation of fibers in the temporal bone). Laryngoscope 1973;83:1311-29.

10. Bonavolonta G, Tranfa F, de Conciliis C, Strianese D. Dermoid cysts: 16-year survey. Ophthalmic Plast Reconstr Surg 1995;11: 187-92.

11. Samuelson TW, Margo CE, Levy MH, Pusateri TJ. Zygomaticofrontal suture defect associated with orbital dermoid cyst. Surv Ophthalmol 1988;33:127-30.
12. Tsai CC, Lin YC, Kao SC, Kau HC, Hsu WM. Preoperative methylene blue injection as a surgical adjuvant in treatment of orbital dermoid cyst. Acta Ophthalmol Scand 2007;85:909-10.

13. Mucaj S, Ugurel MS, Dedushi K, Ramadani N, Jerliu N. Role of MRI in diagnosis of ruptured intracranial dermoid cyst. Acta Inform Med 2017;25:141-4.

14. Skandalakis JE, Godwin JT, Mabon RF. Epidermoid cyst of the skull: report of four cases and review of the literature. Surgery 1958;43:990-1001.

15. Phelan AL, Jones CM, Ceschini AS, Henry CR, Mackay DR, Samson TD. Sparing a craniotomy: the role of intraoperative methylene blue in management of midline dermoid cysts. Plast Reconstr Surg 2017;139:1445-51. 